Supporting Information

\title{
Optical and morphological properties of single-phased and dual- emissive InP/ZnS quantum dots via transition metallic and inorganic ions
}

Xian Wei ${ }^{1,4}$, Shiliang Mei ${ }^{1,3,4}$, Bobo Yang ${ }^{2}$, Zhihao Chen ${ }^{2}$, Hanqing Dai ${ }^{2}$, Zhe Hu ${ }^{1}$, Guilin Zhang ${ }^{1}$, Fengxian Xie ${ }^{1}$, Wanlu Zhang ${ }^{1, *}$, Ruiqian Guo ${ }^{1,2, *}$

1 Engineering Research Center of Advanced Lighting Technology, Ministry of Education; Institute for Electric Light Sources, Fudan University, Shanghai 200433, China

2 Institute of Future Lighting, Academy for Engineering and Technology, Fudan University, Shanghai 200433, China

3 Joint Key Laboratory of the Ministry of Education, Institute of Applied Physics and Materials Engineering, University of Macau, Avenida da Universidade, Taipa, Macau 999078, China 4 Authors contributed equally.

* Corresponding author.

Tel.: +86 2155664588.

E-mail address: rqguo@fudan.edu.cn, fdwlzhang@,fudan.edu.cn

\begin{tabular}{|l|l|}
\hline Number of pages: & 7 \\
\hline Number of schemes: & 0 \\
\hline Number of figures: & 5 \\
\hline Number of tables: & 3 \\
\hline
\end{tabular}


This file includes:

1. Elemental analysis (ICP) data for the $\mathrm{InP} / \mathrm{ZnS}, \mathrm{Cu}: \mathrm{InP} / \mathrm{ZnS}, \mathrm{Ag}: \mathrm{InP} / \mathrm{ZnS}$ and $\mathrm{Mn}: \mathrm{InP} / \mathrm{ZnS}$ QDs. (Table S1)

2. Decay times, amplitude constant ratios of PL emission and absolute PL QYs for three doped InP/ZnS QDs. (Table S2-S3)

3. TEM and HRTEM images of four kinds of InP cores and Ag:InP QDs with different precursors. (Figure S1-S2)

4. SAED patterns of SAED patterns of (a) InP/ZnS, (b) $\mathrm{Cu}: \operatorname{InP} / \mathrm{ZnS}$, (c) Ag:InP/ZnS and (d) Mn:InP/ZnS QDs. (Figure S3)

5. High resolution spectra of In3d, Zn 2p, S 2p and P 2p of (a) InP/ZnS, (b) Cu:InP/ZnS, (c) $\mathrm{Ag}: \operatorname{InP} / \mathrm{ZnS}$ and (d) Mn:InP/ZnS QDs. (Figure S4-S5) 
Table S1 The elemental analysis (ICP) data for the InP/ZnS, Cu:InP/ZnS, Ag:InP/ZnS and Mn:InP/ZnS QDs.

\begin{tabular}{cccccc}
\hline Net weight (mg/kg) & In & P & Zn & S & Dopant \\
\hline InP & 418.3 & 104.8 & $/$ & $/$ & $/$ \\
Cu:InP & 158.9 & 10.0 & 225.9 & 75.7 & 2.1 \\
Ag:InP & 98.7 & 5.1 & 153.8 & 44.6 & 5.1 \\
Mn:InP & 90.8 & 22.2 & 140.6 & 35.8 & 37.3 \\
\hline
\end{tabular}

Table S2 Decay times and amplitude constant ratios of PL emission for $\mathrm{Cu}: \mathrm{InP} / \mathrm{ZnS}$, Ag:InP/ZnS and Mn:InP/ZnS QDs. 


\begin{tabular}{ccccccc}
\hline & $\boldsymbol{\lambda}(\mathbf{n m})$ & $\mathbf{B}_{\mathbf{1}} / \%$ & $\boldsymbol{\tau}_{\mathbf{1}}$ & $\mathbf{B}_{\mathbf{2}} / \%$ & $\boldsymbol{\tau}_{\mathbf{2}}$ & $\boldsymbol{\tau}_{\mathrm{av}}$ \\
\hline \multirow{3}{*}{ Cu:InP/ZnS QDs } & 510 & $74 \%$ & $44.8 \mathrm{~ns}$ & $26 \%$ & $177.5 \mathrm{~ns}$ & $122 \mathrm{~ns}$ \\
\cline { 2 - 7 } & 581 & $40 \%$ & $46.9 \mathrm{~ns}$ & $60 \%$ & $260.7 \mathrm{~ns}$ & $237.8 \mathrm{~ns}$ \\
\hline \multirow{2}{*}{ Ag:InP/ZnS QDs } & 501 & $77 \%$ & $44 \mathrm{~ns}$ & $23 \%$ & $121.8 \mathrm{~ns}$ & $79.2 \mathrm{~ns}$ \\
\cline { 2 - 7 } & 582 & $23 \%$ & $76.5 \mathrm{~ns}$ & $77 \%$ & $250.7 \mathrm{~ns}$ & $236.1 \mathrm{~ns}$ \\
\hline \multirow{3}{*}{ Mn:InP/ZnS QDs } & 508 & $62 \%$ & $17 \mathrm{~ns}$ & $38 \%$ & $68.9 \mathrm{~ns}$ & $54 \mathrm{~ns}$ \\
\cline { 2 - 7 } & 584 & $87.5 \%$ & $0.179 \mathrm{~ms}$ & $12.5 \%$ & $3.14 \mathrm{~ms}$ & $2.29 \mathrm{~ms}$ \\
\hline
\end{tabular}

Table S3 Absolute PL QYs of three kinds of doped InP QDs

\begin{tabular}{cccc}
\hline QDs & Cu:InP/ZnS & Ag:InP/ZnS & Mn:InP/ZnS \\
\hline PL QY & $75.8 \%$ & $91.04 \%$ & $50.8 \%$ \\
\hline
\end{tabular}




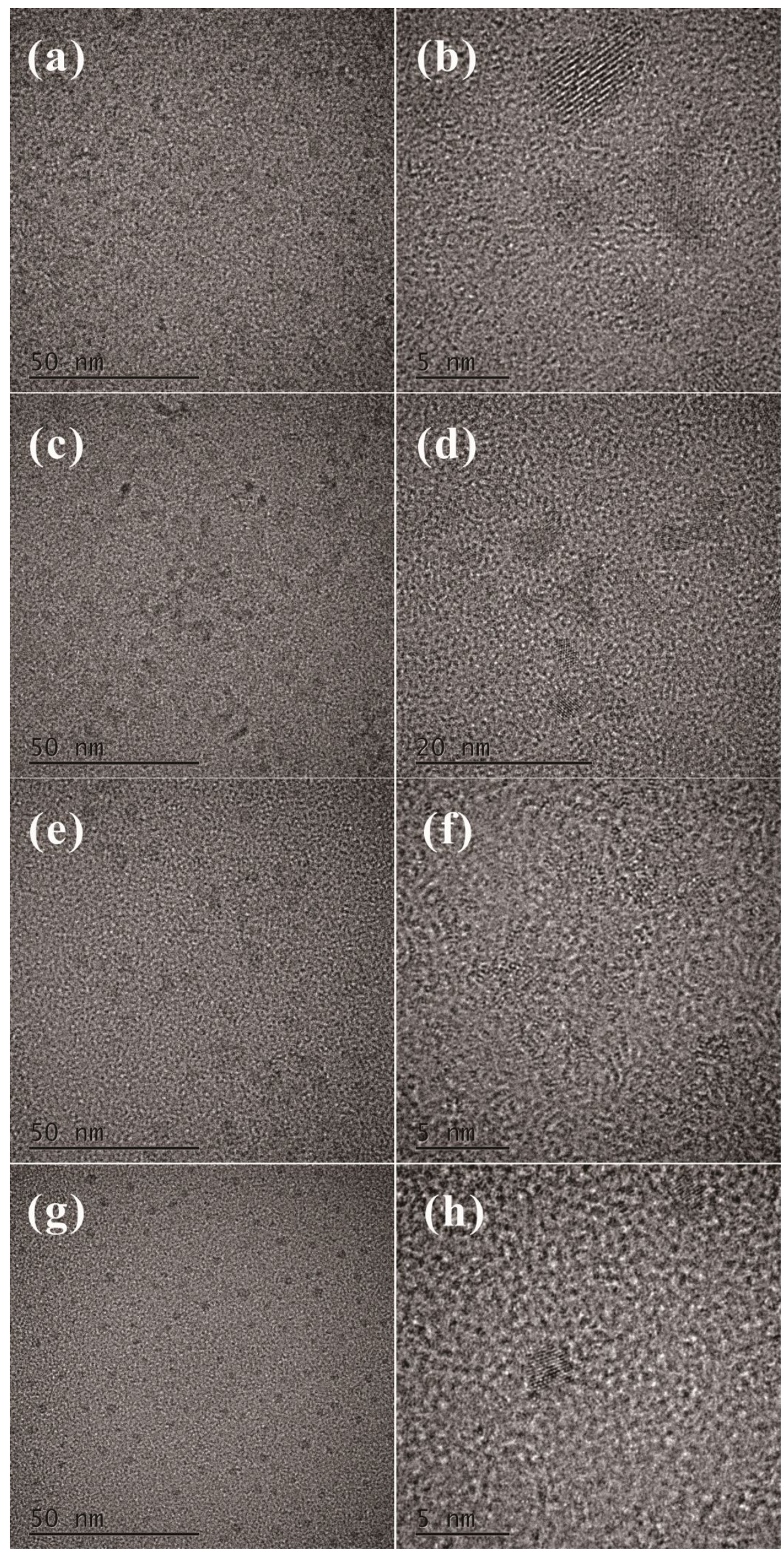


Fig. S1 TEM and HRTEM images of (a, b) InP, (c, d) Cu:InP, (e, f) Ag:InP and (g, h) Mn:InP QDs.

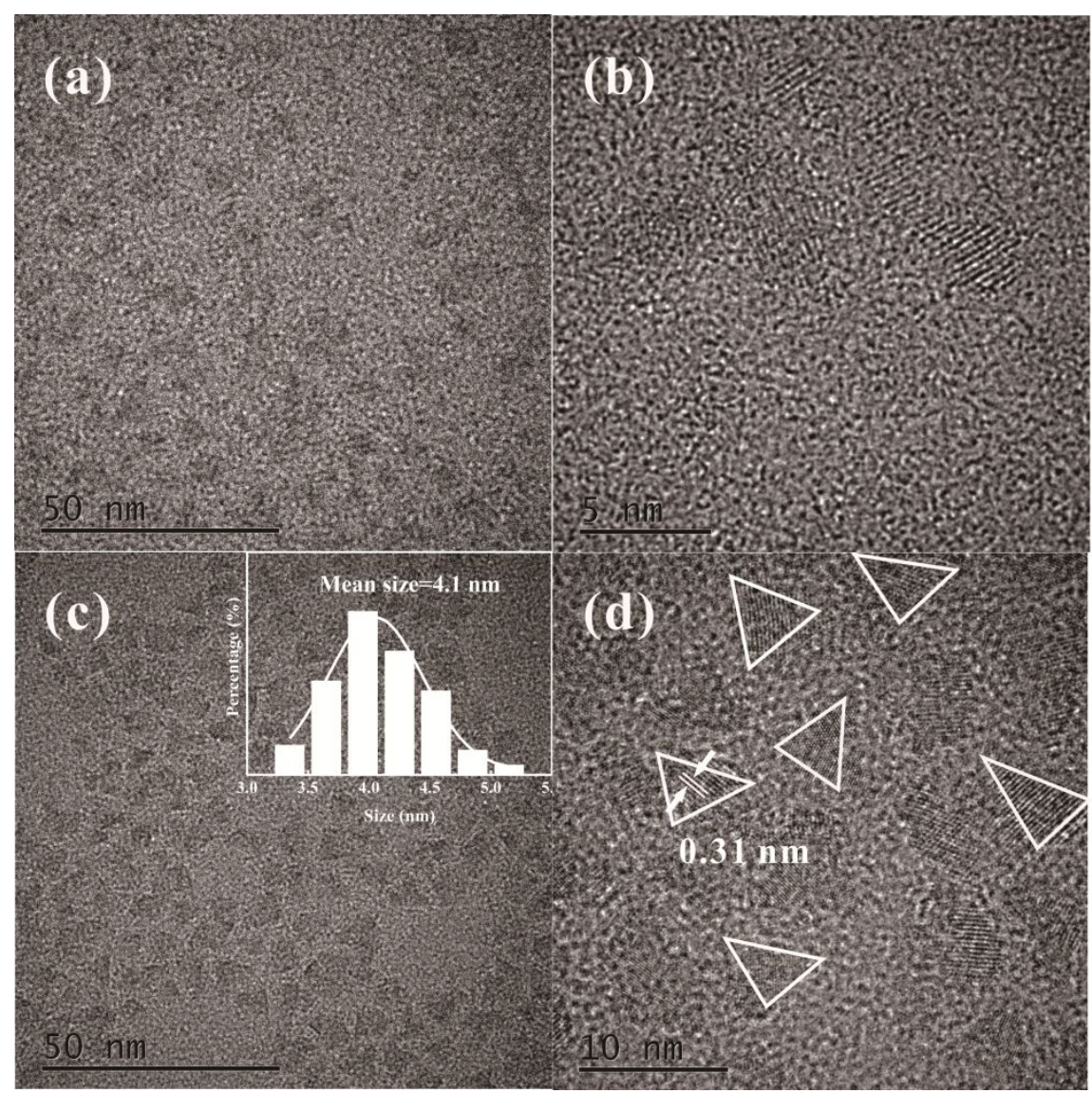

Fig. S2 (a) TEM and (b) HRTEM images of Ag:InP core ( $\mathrm{AgCl}$ precursor), (c) TEM and (d) HRTEM images of $\mathrm{Ag}: \mathrm{InP} / \mathrm{ZnS}$ QDs ( $\mathrm{AgCl}$ precursor). The inset is size distribution. 


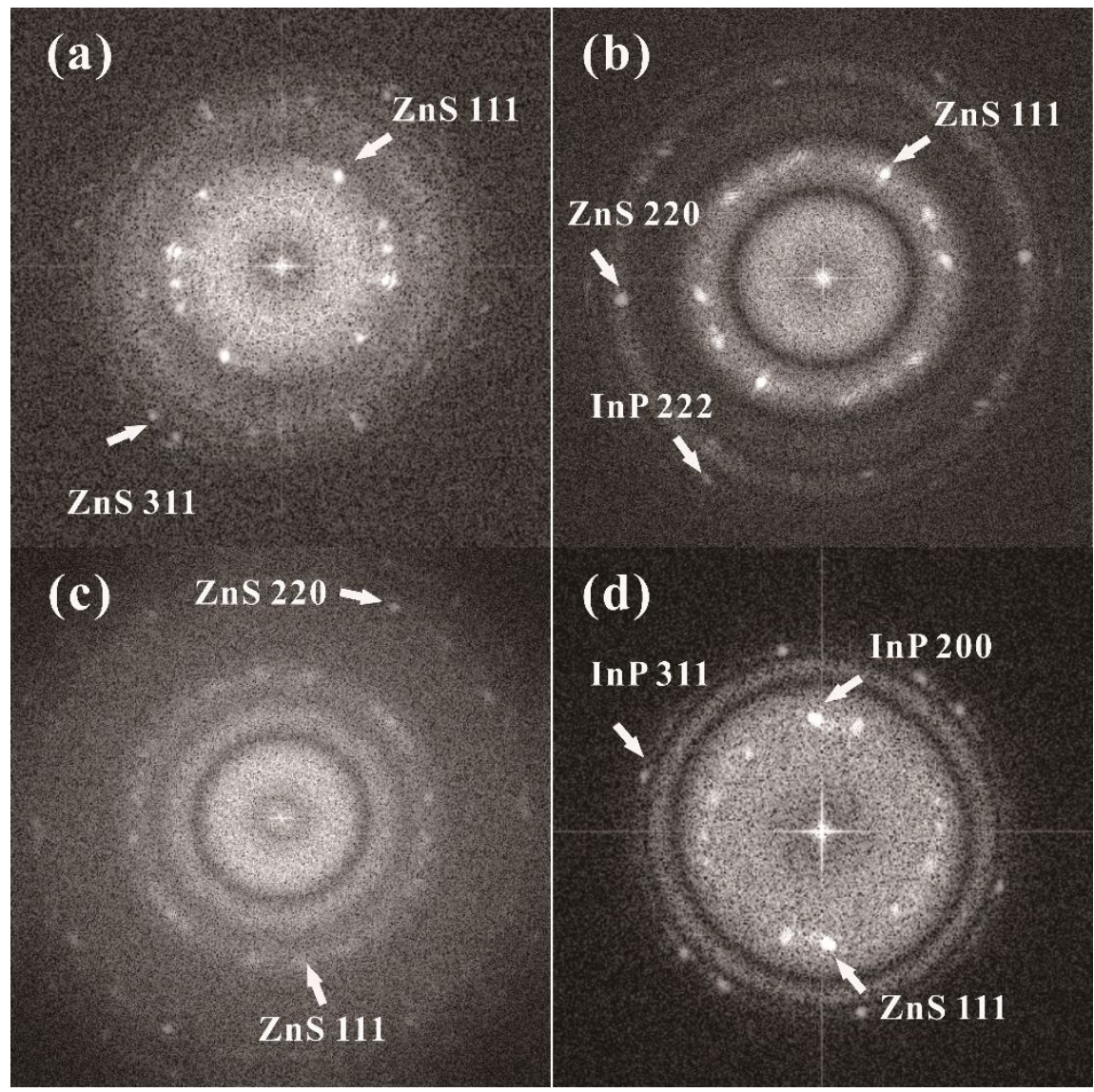

Fig. S3 SAED patterns of (a) InP/ZnS, (b) Cu:InP/ZnS, (c) Ag:InP/ZnS and (d) $\mathrm{Mn}: \operatorname{InP} / \mathrm{ZnS}$ QDs.

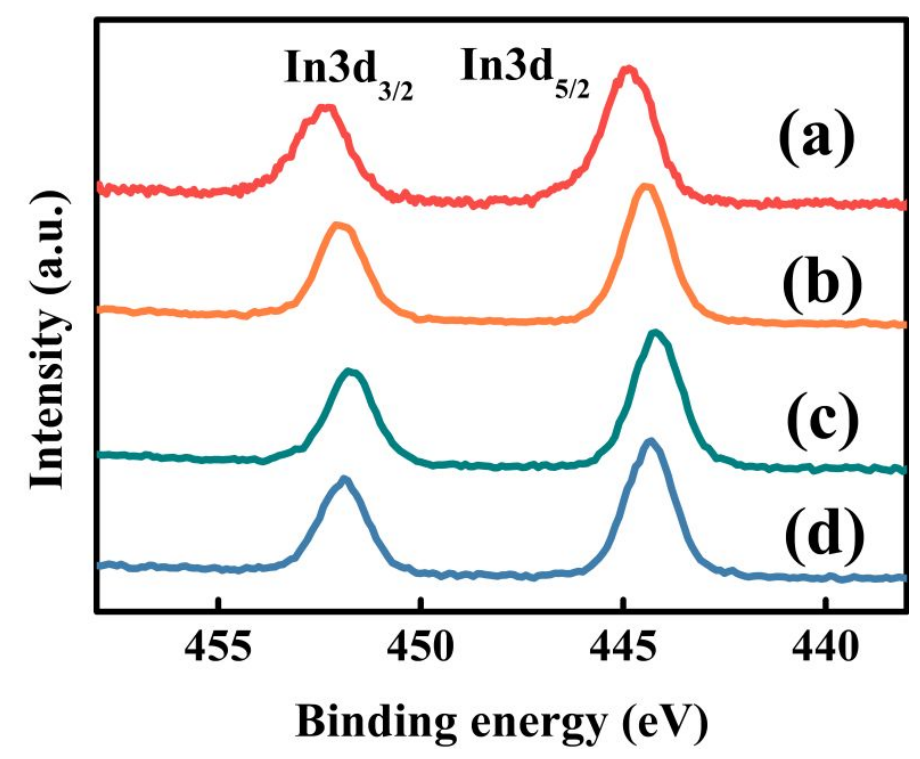

Fig. S4 High resolution In3d spectra of (a) InP/ZnS, (b) $\mathrm{Cu}: \mathrm{InP} / \mathrm{ZnS}$, (c) Ag:InP/ZnS and (d) Mn:InP/ZnS QDs. 


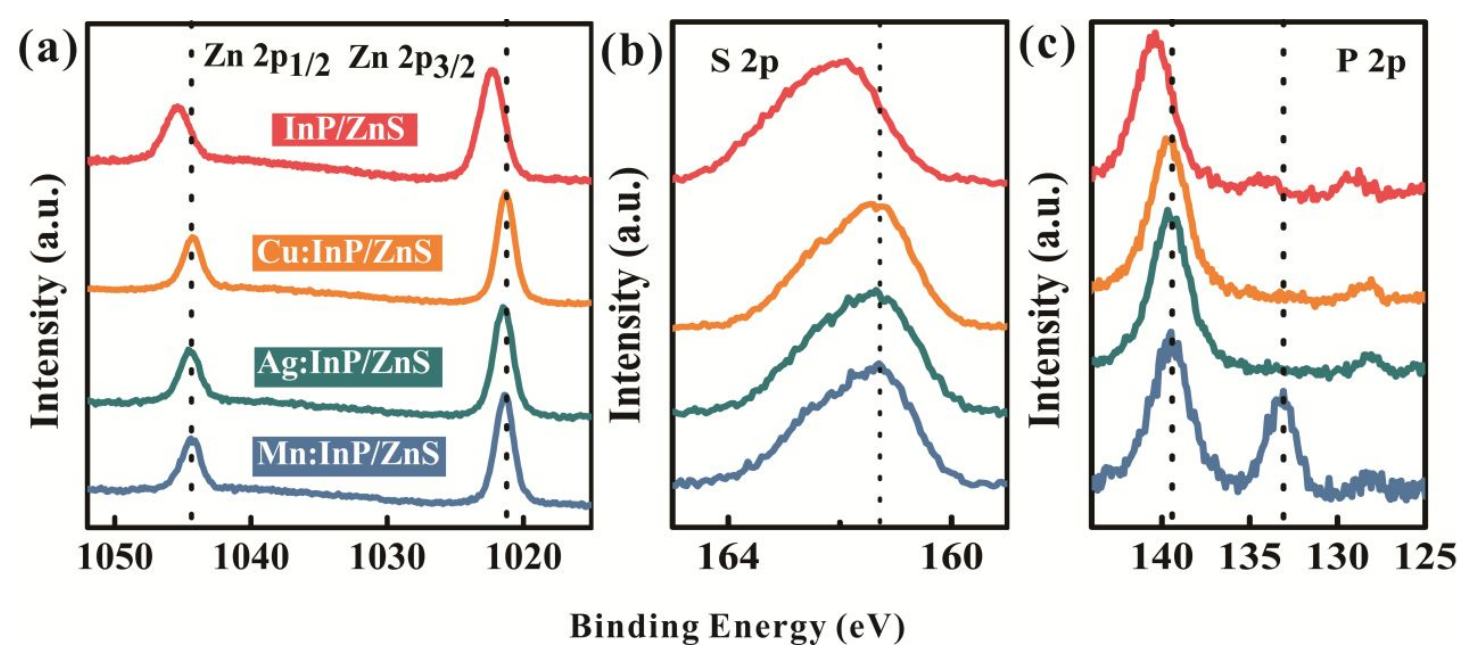

Fig. S5 High resolution spectra of (a) Zn 2p, (b) S 2p and (c) P 2p. 